\title{
17 Hidroxiprogesterona en el Diagnóstico y Tratamiento del Síndrome Adrenogenital
}

\author{
Dr. Fernando Cassorla.
}

\section{Hydroxyprogesterone in the diagnosis of Adrenogenital Syndrone}

\begin{abstract}
We have reviewed the value of the radioimmunoassay for serum 17 hydroxyprogesterone in the diagnosis and management of children with the 21 hydroxylase deficiency form of congenital adrenal hyperplasia. The usefulness of this assay in the diagnosis of allected newborns has been highlighted. Special reference is made regarding the characteristics of the adrenal gland netabolism during the neonatal period. Intormation is provided on the hydroxyprogesterone levels in heteruzygote carriers, and in patients with the acquired form of the disease. In addition, we have examined the value of the assay in the management of patients with this disorder by describing some illustrative cases.

It is conchuded that the radioimmunoassay for serum 17 hydroxyprogesterone represents a significant advance in the diagnosis of patients with the 21 hydroxylase deficiency fonm of congenital adrenal hyperplasia. The assay is ot less value in the management of patients with this condition.
\end{abstract}

El sindrome adrenogenital causado por hiper. plasia suprarrenal está caracterizado por la presencia de un déficit enzimático que limita la producción de hormonas esteroidales. Con menor frecuencia este síndrome puede ser producido por un tumor suprarrenal. En este artículo nos referiremos sólo a aquellas variedades del sindrome caracterizadas por un déficit enzimático en la glándula suprarrenal. Estas fonmas constituyen un error innato del metabolismo que se transmite a través de un gen autosómico recesivo.' Las formas más comunes del sindrome están caracterizadas por virilización, que es causada por la excesivat producción de andrógenos por la corteza suprarrenal, ${ }^{2-4}$ si bien existen otras variedades que no estín asociadas con este problema. ${ }^{x-9}$ Algunas características de las fornas más comunes del sîndrome se presentan en la Tabla 1.

Clásicumente, el diagnóstico y tratamiento del síndrome adrenogenital ha estado basado en la determinación de los 17 cetosteroides y pregnanetrial urinario. ${ }^{10}$ Debido a que la cortez: suprarrenal secreta honnonas con un marcado ritno

\footnotetext{
Irnvestizador Ascriado. Development Endocrinolong Branch Xational Iustitute of Clild Health ind Human Decelopment, National Institutes of Health, Bethesdd, Maryland, USA.
}

Tabla 1

C] asificacion del sindrome adrenogenital assceiado con un déficit en la producción de cortisol.

\begin{tabular}{|c|c|c|c|}
\hline $\begin{array}{l}\text { Deficielrcia } \\
\text { enzináticäa }\end{array}$ & $\begin{array}{l}\text { Virili- } \\
\text { zación }\end{array}$ & $\begin{array}{l}\text { Pérdida } \\
\text { de sal }\end{array}$ & $\begin{array}{l}\text { Hiper- } \\
\text { tensión }\end{array}$ \\
\hline 20,22 destuolatida & - & + & - \\
\hline 17 hidruxilasa & - & - & + \\
\hline 3 B-ol deshidrogenasa & \pm & $\pm t^{b}$ & - \\
\hline 11 hidroxilasa & + & - & $+c$ \\
\hline 21 hidroxilasa & + & $+^{\text {h }}$ & - \\
\hline
\end{tabular}

a Las ninas tienen una virilización modesta y los vasones estan masculinizados en forma ínsuficiente.

bExisten casos perdedores de sal y otros compensados. "Hxisten formas hipertensivas y ot as nomotensivas.

circadiano, caracterizado por niveles altos en la madrugada y bajos en la tarde, la excreción trinaria de estos productos hormonales ha permitido una visión integrada de la función de esta glándu1a. Sin embargo, este es un método relativamente poco sensible para la determinación de la función suprarrenal, y tiene además el inconveniente de requerir colecciones de orina que pueden constituir un problema en niños sin control esfinteriano. 
El desarrollo de la téenica del radioinmunoanálisis ha incrementado en forma significativa nuestra capacidad para medir la concentración plasmáticu de diferentes hormonas, con el consiguiente avance en el diagnóstico y tratamiento de diversas enfermedades endocrinas. En este articulo se hace un analisis del progreso que ha significado la aplicación del radioinmunoanálisis para medir la 17 hidroxiprogesterona en el diagnóstico y tratamiento del sindrome adrenogenital. Para simplificar esta discusión nos referiremos en particular a la forma más común del síndrome, aquella por déficit de la enzima 21 hidroxilasa, si bien los conceptos fundamentales expresados en este artículo son extrapolables a algunas de las otras formas del mismo.

\section{Metabolismo suprarrenal}

Para poder entender los avances que han ocurrido en este campo, es necesario hacer una breve reseña del metabolismo de la corteza suprarrenal. Un esquema simplificado del metabolismo esteroidal es presentado en la Figura 1. Esta figura ilustra en forma esquemática el proceso metabôlico que conduce a la biosintesis de mineralocorti- coides (aldosterona), ylucocorticoides (cortisol), y hormonas sexuales. Como se observa en la Figura 1 , el déficit de 21 hidroxilasa impide la síntesis normal de cortisol y aldosterona, pero no interfiere en la producción de los andrógenos que no requieren de esta enzima para su biosíntesis. El déficit de cortisol induce una producción exagerada de la hormona adrenocorticotópica hipotisiaria (ACTH), que a su vez tiene por función estimular a la glándula suprarrenal. Se establece de este modo un círculo vicioso que conduce a hiperplasia suprarrenal y a la excesiva producción de andrógenos. El círculo vicioso puede ser corregido al instituir tratamiento con cortisol, lo que normaliza la secreción de ACTH, y por consecuencia elimina la exagerada estimulación de la glándula suprarrenal.

El síndrome adrenogenital por deficiencia de 21 hídroxilasa se caracteriza clínicamente por la presencia de insuficiencia suprartenal asociada con vírilización. ${ }^{10}$ Los casos más severos pueden manifestar un cuadro de deshidratación, hiponatremia e hipercaliemia, generalmente durante las primeras semanas de vida. Este cuadro se debe presumiblemente a un déficit en la producción de aldosterona. ${ }^{11,12}$ De no mediar un diagnóstico y
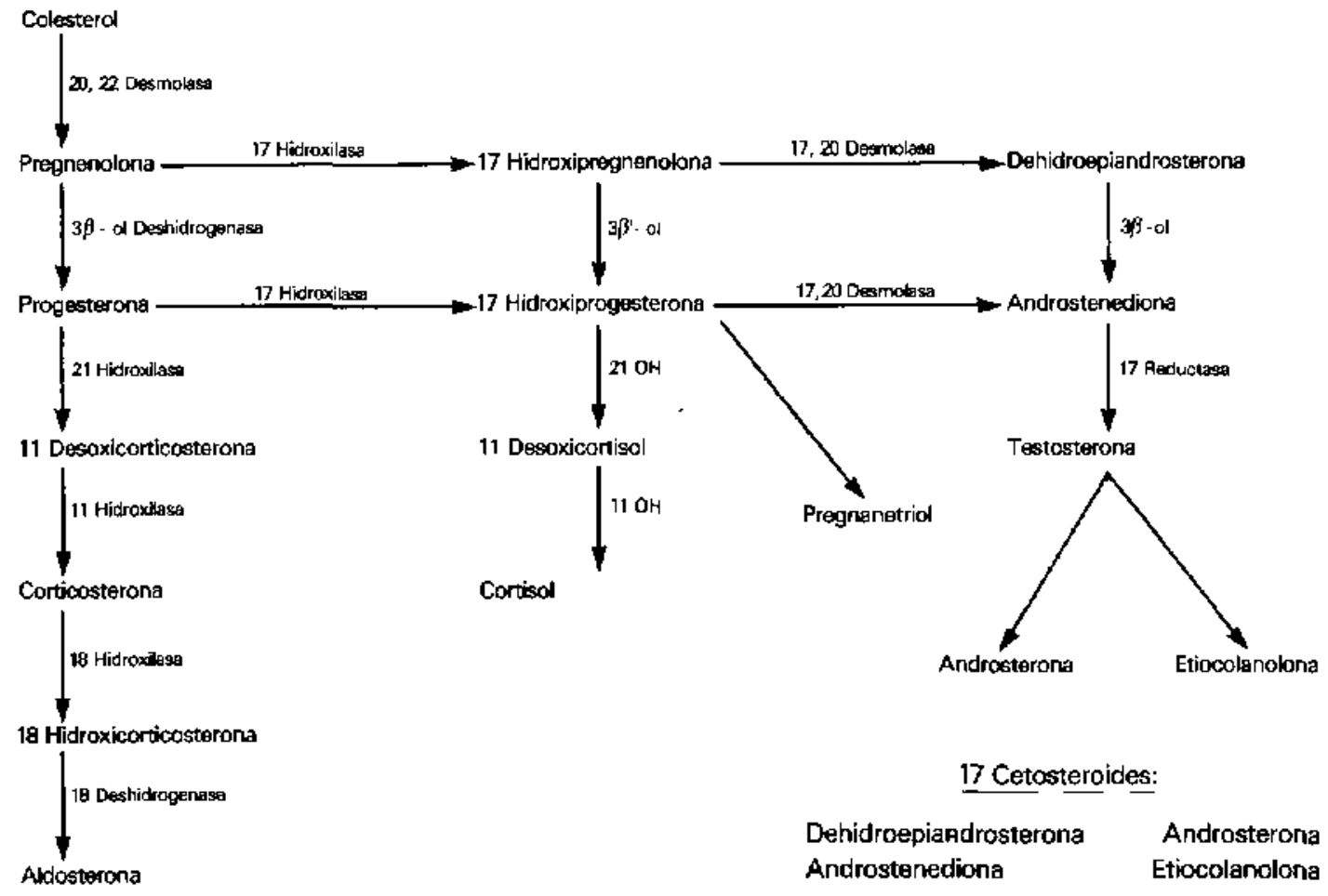

17 Cetosteroides:

Dehidroepiandrosterona Androstenediona

Androsterona Etiocolanolona

Figura 1. Esquema simplificado del metabolismo suprartenal. 
tratamiento oportuno en estos casos, el desenlace puede ser fatal.

El diagnóstico del síndrome adrenogenital es confirmado por un aumento en el nivel de los 17 cetosteroides urinarios. Algunos de estos compuestos están ilustrados en forma esquemática en la Figura 1, y como se observa, ellos comprenden a la mayoría de los esteroides sexuales de origen suprarrenal. Como su nombre lo indica, estos compuestos tienen un grupo cetona en posición 17 y son detectados por la sencilla reacción colorimétrica de Zimmermann. ${ }^{13}$

EI otro compuesto de gran utilidad para el diagnóstico del sîndrome adrenogenital es el pregnanetriol. La concentración urinaria de este esteroide puede ser medida por el inétodo descrito por Bongiovanni. ${ }^{14}$ Como se observa en la Figura 1, este compuesto es el producto de excreción renal de la 17 hidroxiprogesterona. Es obvio que en el sindrome adrenogenital por deficiencia de 21 hidroxilasa existirá una acumulación del metabolito más próximo al bloqueo enzimático, en este caso la 17 hidroxiprogesterona, $y$ de su producto de excreción renal, el pregnanetriol. ${ }^{15}$ Por este motivo, la presencia de niveles elevados de 17 cetosteroides y pregnanetriol urinario proponciona la confirnación diagnóstica del sindrome adrenogenital por deficiencia de 21 hidroxilasa.

Diagnsistico en el recién nacido basado en los 17 cetosteroides y el pregnanetriol urinario.

Este cuadro, aparentemente tan claro, es complicado por el hecho de que durante las primeras semanas de vida la glándula supranenal tiene características bastante diferentes a aquellas descritas previamente. Junto a las clásicas zonas glomerulosa, fasciculata y reticular, la corteza supranenatl del recién nacido posee una exterısa zona denominada fetal. Esta zona tiene como característica bioquímica principal una deficiencia relativa en la enzima $3 \mathrm{~B}$-ol deshidrogenasa. ${ }^{16}$ La zona fetal posee además otras características que no es propio analizar en un articulo de esta naturaleza. La Figura 1 pennite observar gráficamente las consecuencias de este déficit fisiológico de la enzima 3 B-ol deshidrogenasa durante las primeras semanas de vida.

En el recién nacido nomal, la acumulación de dehidroepiandrosterona y de otros 17 cetosteroides determina niveles relativamente elevados de estos compuestos durante el periodo neonatal. ${ }^{17}$
En el recién nacido afectado con el síndrome adrenogenital por deficiencia de 21 hidroxilasa, la elevación del pregnanetriol generalmente demora algunos días en manifestarse $\mathrm{e}^{18,19}$ y solo demuestra una elevación patológica después de la segunda semana de vida. Ello parece deberse a limitaciones en el metabolismo de la 17 hidroxiprogesterona a pregnanetriol durante este período. ${ }^{18}$ En otras palabras, durante el períudo más crítico para establecer un diagnóstico preciso del sindrome adrenogenital, que es cuando el recién nacido tiene el mayor riesgo de manifestar una crisis perdedora de sal, el diagnóstico basado en la determinación de los 17 cetosteroides y pregnanetriol urinario puede ser potencialmente dificil.

La tendencia del recién nacido nonrial a tener niveles relativamente elevados de 17 cetosteroides, y la tendencia de todos los recién nacidos, incluso acueilos con el síndrome adrenogenital, a tener niveles relativamente bajos de pregnanetriol, puede interferir con nuestra capacidad para establecer este diagnóstico basado en la determinación de los 17 cetosteroides y el pregnanetriol urinario. Esto tiene particular importancia para los recién nacidos varomes corl este sindrome, ya que su virilización puede tardar algunos meses en manifestarse por lo que el diagnóstico clínico puede ser difícil.

\section{Diaynóstico en el recién nacido basado en la 17} hidroxiprogesterona.

El radioinmunoanálisis para la 17 hidroxiprogesterona ha aumentado en forma significativa nuestra capacidad para medir la concentración plasmática de este compuesto. ${ }^{.00}$ La alta sensibilidid de este métudo permite deteminar concentraciones de este esteroide que no pueden ser detectados por el método colorimétrico que mide su producto de excreción renal, el pregnanetriol. ElIo es demostrado en la Tabla 2 que indica las concentraciones plasmáticas de 17 hidroxiprogesterona y de pregnanetriol urinario en 4 recién nacidos con el síndrome adrenogenital por deficiencia de 21 hidroxilasa. La Tabla 2 indica una elevación patológica en los niveles de 17 hidroxiprogesterona en todos los pacientes, aún cuando el pregnanetriol urinario no es detectable en 3 de los 4 casos.

La determinación de 17 cetosteroides urinarios también puede ser de poca utilidad diagnóstica durante este período, debido a la tendencia del 
17 hidroxiprogesterona plasmática $y$ pregnanetriol urinario en 4 recién nacidos con el síndrome adrenogenital por deficiencia de 21 hidroxilasa

\begin{tabular}{|c|c|c|c|}
\hline Paciente & $\begin{array}{l}\text { Edad } \\
\text { (días) }\end{array}$ & $\begin{array}{c}17 \text { hidroxiprogesterona } \\
\text { (ngidl) }\end{array}$ & $\begin{array}{l}\text { Pregnanetriul } \\
\quad \text { (mg/24h) }\end{array}$ \\
\hline J.H. & 4 & .32 .300 & Nodetectable \\
\hline B.S. & 7 & 27.600 & Nudetectable \\
\hline M.R. & 10 & 41.700 & No detectable \\
\hline G.H. & 19 & $18.800)$ & 0,23 \\
\hline $\begin{array}{l}\text { Nivel fisiológico } \\
\text { (recién nacidos) }\end{array}$ & & $<200$ & $<0,10$ \\
\hline
\end{tabular}

recién nacido nonnal a tener niveles relativamente elevados de estos esteroides. ${ }^{[7}$ Por este motivo, la concentración de 17 cetosteroides en recién nacidos norrnales y en aquellos afectados con el síndrome adrenogenital pueden ser ocasionalmente indistinguibles. ${ }^{10}$

Por el contrario, los niveles plasmáticos de 17 hidroxiprogesterona en los pacientes con el sindrome adrenogenital son muy superiores ad nivel fisiológico. ${ }^{21,} 22$ Una ventaja adicional en la utilización de 17 hidroxiprogesterona para el diagnóstico del sindrome adrenogenital es que la concentración de este esteroide es relativamente constante desde el nacimiento hasta la nadurez.$^{2.3}$ Por este notivo los problemas inherentes en establecer un diagnóstico preciso del síndrome adrenogenital durante las primeras semanas de vida son obviados al medir la concentración plasmática de 17 hidroxiprogesterona.

Los niveles de este esteroide pueden variar desde aproximadamente 6.000 hasta $80.000 \mathrm{ng} / \mathrm{dl}$ en pacientes con el síndrome adrenogenital. ${ }^{23-25}$ Esto se compara con un nivel fisiológico de aproximadamente $200 \mathrm{ng} / \mathrm{dl}$. En otras palabras, la gran mayoría de pacientes con el sindrome adrenogenital tiene concentraciones de 17 hidroxiprogesterona al menos 30 veces superiores al nivel fisio lógico de este esteroide. Este método es tan sensible, que algunos investigadores han podido establecer el diagnóstico del sindrome durante la vida fetal al medir la concentración de 17 hidroxiprogesterona en el liquido amniótico. ${ }^{26}$ Recientemente se ha instituido un programa piloto para la detección del síndrome adrenogenital en recién nacidos que pertenecen a grupos étnicos con una alta incidencia del sindrome. ${ }^{2 \pi}$ Este programa está basado en la determinación de 17 hidroxiprogesterona en muestras de sangre obtenidas en papel filtro. ${ }^{24}$ Ello refleja la notable simplificación en el diagnóstico de este síndrome que ha significado la introducción del radioirumunoanálisis para la 17 hidroxiprogesterona.

Diagnóstico del sindrome adrenogenital adquirido

Junto a las clásicas fomas del síndrome adrenogenital cue se manifiestar durante las primeras semanas de vida, existen otras de presentación más tardia. Estos pacientes, que son habitualmente varones, por lo que su detección clínica es algo más difícil, manifiestan el característico crecimiento acelerado y virilización durante la niñez. ${ }^{10}$ El diagnóstico puede establecerse en estos casos por la determinación de los 17 cetosteroides y pregnanetriol urinario.

Diversos autores han descrito adolescentes y adultus jóvenes con manifestacioneśaún más sutiles del síndrome adrenogenital, que consisten principalmente en hirsutismo, trastornos menstruales o infertilidad en la mujer, ${ }_{2}^{29-31}$ y al menos en un caso, aumento del volumen testicular en el varón. ${ }^{32}$ Estos pacientes no tienen evidencia de virilización más marcada, crecimiento acelerado durante la niñez, o fusión prematura de las epítisis óseas, lo que los distingue de los enfermos más típicos con el síndrome. Estos casos han sido denominados "adquiridos" aunque la evidencia indica, con una excepción, ${ }^{33}$ que ellos constihyen una misma enfermedad genética con las formas 
clásicas del síndrome adrenogenital. ${ }^{29,34}$ Es probable que en estos pacientes el déficit enzimático esté presente desde la vida intrauterina, si bien la expresión clínica del síndrome sea más sutil debido a que la deficiencia enzimática es presumiblemente de menor magnitud.

Los pacientes con el síndrome adquirido generalmente manifiestan una modesta elevación de los 17 cetosteroides y pregnanetriol urinario, ${ }^{29-31}$ por lo que estas determinaciones pueden ser de poco valor diagnóstico. Sin embargo, las concentraciones plasmáticas de 17 hidroxiprogesterona son de gran utilidad al revelar niveles claramente patologicos de este esteroide. ${ }^{29-31}$ En algunos de estos pacientes, el déficit enzimático es tan mínimo que solo puede ser diagnosticado durante la estimulación de la glándula suprarrenal con la hormona adrenocorticotópica hipotisiaria (ACTH). Los niveles basales de 17 hidroxiprogesterona pueden ser normales, pero ellos aumentan en forma patológica durante la estimulación suprarrenal. Ello refleja la relativa incapacidad de la enzima 21 hidroxilasa para convertir 17 hidroxiprogesterona en desoxicortisol durante la estimulación suprarrenal, cuando se dispone proporcionalnıente de más sustrato para la biosíntesis esteroidal (Fig. 1).

Este concepto es demostrado gráficamente en la Figura 2 que indica los niveles de 17 hidroxiprogesterona durante la estimulación suprarrenal en enfermos con diversas formas del síndrome adrenogenital. La figura indica que los pacientes con el síndrome adquirido tienen niveles de $\mathbf{1 7}$ hidroxiprogesterona que son intermedios entre aquellos observados en la forma clásica de síndrome y en los portadores heterocigotos.

La descripción de diversos casos genuinos del sindrome adrenogenital adquirido demuestra que esta entidad, cuya existencia ha sido cuestionada por algunos autores, ${ }^{35}$ constituye una entidad clinicat real. Algunos de estos casos corresponden a las otras formas enzimáticas del síndrome, ${ }^{36-36}$ indicando que no tan sólo el déficit de 21 hidroxilasa puede manifestarse como sindrome adquirido. La heterogeneidad en las manifestacioner clínicas del síndrome adrenogenital probablemente refleja variaciones en el carácter del déficit enzimático presente en los diferentes casos. Este amplio espectro en las manifestaciones clínicas ha sido descrito en otros errores innatos del metabolismo caracterizados por una deficiencia enzimática.

Lal expansión de nuestro concepto de la entidad

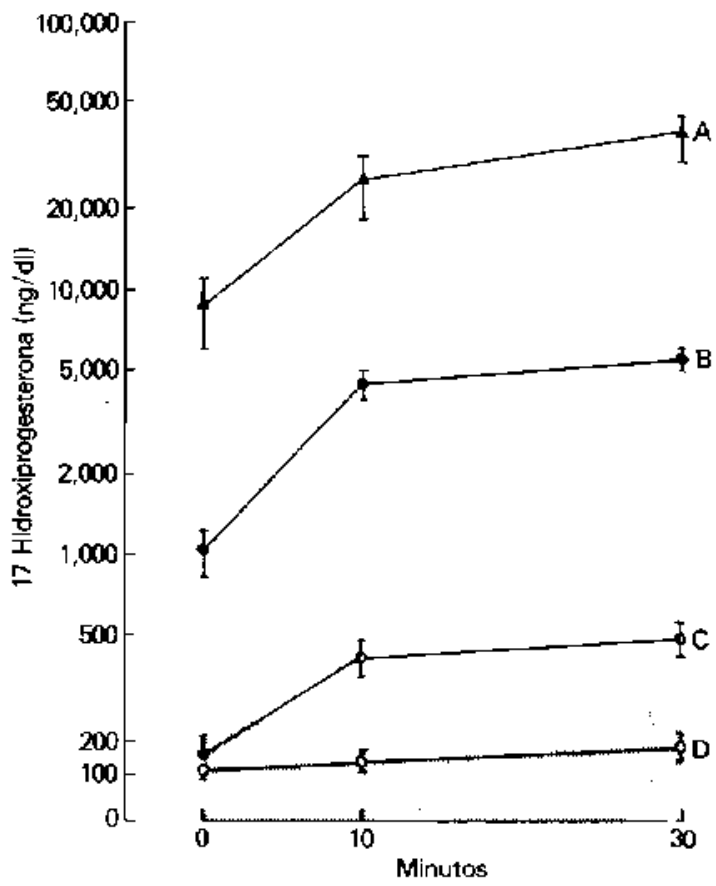

Figura 2, Concentraciones plasmáticas de 17 hidroxiprogesterutia durante la estimulación suprarsenal con ACTH (promedioy cror standard). A representa pacientes con el sindrome adrenogenital clasico (n = 3), B pacientes con el sindrome adquirido ( $n=8$, C portadores heterucigotos $\{n=10$, y $D$ el gnipo sontmol (n = 11). Notese la escala logarítmica ел la ordenada. La zona gris represtenta el rango northal.

clínica representada por el síndrome adrenugenital tiene una importante connotación terapéutica, debido a que los pacientes con la forma adquirida del síndrome manifiestan una mejoria de sus sintomas al ser tratados con glucocorticoides. . $^{24,31,32}$ ${ }^{37}$ Ello es significativo cuando se considera que algunos autores han estimado la prevalencia del síndrome adquirido en alrededor del $9 \%$ de los pacientes con hirsutisıno." Sin embargo, las estimaciones sobre prevalencia varían nutablemente dependiendo de la serie que se considere. En todo caso, el síndrome adrenogenitil adquirido debe ser considerado en el diagnóstico diferencial de las pacientes con hirsutismo, y esta posibilidad diagnóstica debe ser investigada con determinaciones de 17 hidroxiprogesterona tanto en el estado basal como durante la estimulación suprarrenal.

\section{Diagnóstico de los portadores heterocigotos}

E] sindrome adrenogenital por deficiencia de 21 hidroxilasa es transmitido por un gen autosí- 
mico recesivo, ${ }^{1}$ que está en estrecha asociación con el sistena de histocompatibilidad HLA ${ }^{34} \mathrm{Di}$ versos investigadores han intentado describir algún método para diagnosticar a los portadores heterocigotos del sindrome con el fin de identificar it aquellas parejas con mayor riesgo de tener un niño afectado. Estos esfuerzos han estado basados en el concepto de (jue por una parte, los heterocigotos compaten la mitad de haplotipo HLA con los homocigotos, y por otra de que ellos manifiestan evidencia de un mínimo grado de deficiencia enzinuítica.

La existencia de un déficit enzimático no ba sido demostrada en funma consistente en los portadores con la determinación de los 17 cetosteroides y el pregnanetriol urinario. ${ }^{40-42}$ Con el advenimiento del radioinmunoanálisis para medir la 17 hidroxiprogesterona, ammentaron las expectativas de poder demostrar un bloxpeo enzinatico en estos pacientes. Diversos estudios llevados a cabo durante la estimulación suprarrenal, han indicado que los niveles de 17 hidroxiprogesteronat, ${ }^{4-45}$ y de otros esteroides, ${ }^{46}$ penniten diagnosticar aproximadamente $2 / 3$ de los heterocigotos.

Las concentraciones de 17 hidroxiprogesterona durante la estimulación suprarrenal en um grupo de portadores se ilustan en la Figura 2. La figura indica que ellos tienen niveles de este esteroide que son intermedios entre aquellos observados en los pacientes con el síndrome adrenogenital adquirido y el grupo control. Es importante enfatizar que un porcentaje significativo de heterocigotos tiene niveles de 17 hidroxiprogesterona que son indistinguibles de la población normal, ${ }^{4 \cdot 3-45}$ por lo que este sistema para identificar portadores tiene sólo una utilidad relativa. Sin embargo, este método representa un avance sustancial cuando se Io compara con el bajo porcentaje de heterocigotos que se pueden identificar con la determinación de los 17 cetosteroides y el pregnanetriol urinario. ${ }^{40-42}$

Dupont ha descrito una estrecha asociación entre el gen que deternina el síndrome adrenogenital por deficiencia de 21 hidroxilasa y el sistenu HLA. Dentro de carla familia los homocigotos poseen un haplotipo HLA que es idéntico, y los portadores comparten la mitad de este haplotipo con los homocigotos..$^{39.47} \mathrm{La}$ identificación de los portadores por este método requiere que se determine el tipo de HLA en ambos progenitores $y$ en el paciente afectado. Esto se debe a que los homocigotos tienen tipos HLA que son idênticoss dentro de cada familia, pero que varían de familia a familia. Este método ha demostrado ser de gran utilidad para clasificar en forma precisa a los diferentes miembros de las tamilias afectadas, y guarda una estrecha relación con el grado de deficiencia crizimática determinado en cada paciente por Ia 17 hidroxiprogesterond. ${ }^{4}$

Recientemente se han descrito pacientes que son homocigotos en base a su tipo HLA, pero que sólo manifiestan un grado intermedio de déficit enzimático. Estos pacientes, denominados cripti$\cos ^{25}$ debido a su completa ausencia de síntomas, no tienen ninguna evidencia de virilización. La descripción de estos pacientes ha contribuido a expandir nuestro concepto del sindrome adrenogenital, y representa otra cemostración del progreso que ha ocurrido en este campo con la introducción del radioinmunoanálisis para la 17 hidroxiprogesterona $y$ de la tipología HLA.

\section{li hidroxiprogesterona como indice de control terapéutico}

Una vez confirmado el diagnóstico del síndrome adrenogenital debe iniciarse el tratamiento con glucocorticoides y en caso necesario con ruineralocorticoides. ${ }^{10}$ El objetivo del tratamiento es: adninistrar una dosis fisiológyca de glucocorticoides para nonnalizar la excesiva secreción de hormona adrenocorticotrópica hipofisiaria (АС ГН), y por consecuencia reducir la exagerada producción

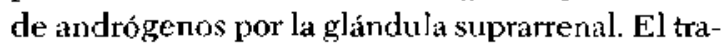
tamiento debe incluir mineralocorticoides si existe un cuadro clínico perdedor de sal, y/o se establece que existe un déficit en la producción de aldosterona.

La definición de lo que constituye una dosis de glucocorticoide apropiada para cada paciente es algo variable, lo gue es demostrado por el amplio rango de dosis sugeridas por diferentes autores. ${ }^{10}$. 49. 50.51 Las dosis recomendadas pueden variar entre aproximadamente 15 y 30 ing de hidrocortisona por $\mathbf{M}^{2}$ de superficie corporal. La eficacia del tratamiento debe controlarse con diversos parámetros, que incluyen el desarrollo pondoestatural, la maduración ósea y algún indice de función suprarrenal. ${ }^{10 .}{ }^{52}$ El riesgo principal de una dosis excesiva de gluccorticoides es el retardo del crecimiento y de la maduración ósea inducida por el tratumiento. El riesgo más signifiçativo de una dosis insuticiente, es la exagerada producción de andrógenos por la glándula suprartenal, lo que 
conduce a una aceleración del crecimiento y de la maduración ósea. Ambos problemas ocurren con cierta frecuencia en las series de pacientes que se han publicado, ${ }^{49,50,53}$

Los índices de función suprarrenal que se controlan más comúnmente durante ef tratamiento del sindrome adrenogenital son los 17 cetosteroides y el pregnanetriol urinario. ${ }^{52}$ Como se indicó previamente, estos metabolitos urinarios tienen la ventaja de proporcionar una visión integrada de la función de esta glándula. Sin embargo, el tratamiento que es basado únicamente en la determinación de estos esteroides puede ser inadecuado. Aún cuando los niveles altos de $\mathbf{1 7}$ cetosteroides y pregnanetriol sugieren que la dosis de glucocorticoide es insuficiente, los niveles nomales de estos esteroides no permiten distinguir entre los pacientes bien controlados y aquellos que están recibiendo una dosis excesiva de glucocorticoide. Ambos grupos de pacientes tienen concentraciones normales de 17 cetosteroides y pregnanetriol, pero los primeros tienen un desarrollo pondoestatural nomnal, mientras los segundos tienen un retraso del crecimiento. ${ }^{51,53}$ Por este motivo, no se debe considerar la normalización de los 17 cetosteroides y el pregnanetriol urinario como el único objetivo del tratamiento, ya que ello generalmente implica recetar dosis excesivas de glucocorticoides. ${ }^{53} \mathrm{El}$ consenso indica que es preferible controlar a los pacientes en edad pediátrica tanto en base a su crecímiento y maduración ósea como por sus niveles de 17 cetosteroides y pregnanetriol urinario. ${ }^{53}$

Diversos autores han intentado establecer si la 17 hidroxiprogesterona, o algún otro esteroide, representa un mejor índice de función suprarrenal para basar el tratamiento del sindrome adrenogenital. $24,24,54,55,56,57,58$ Uno de los problemas en este sentido reside en la interpretación de los niveles de 17 hidroxiprogesterona en relación con el ritmo circadiano suprarrenal, y con las dosis de glucocorticoides administradas a cada paciente. Como resultado de estos estudios, algunos investigadores han cuestionado la correlación entre los niveles de 17 hidroxiprogesterona y otros índices de control en estos pacientes. 56

Un ejemplo representativo de nuestra experiencia, que es similar a la de otros autores, ${ }^{23.57}$ es ilustrada en la Figura 3. Esta figura muestra las concentraciones de 17 hidroxiprogesterona durante el tratamiento de 4 pacientes con el síndrome adrenogenital por deficiencia de 21 hidroxila-

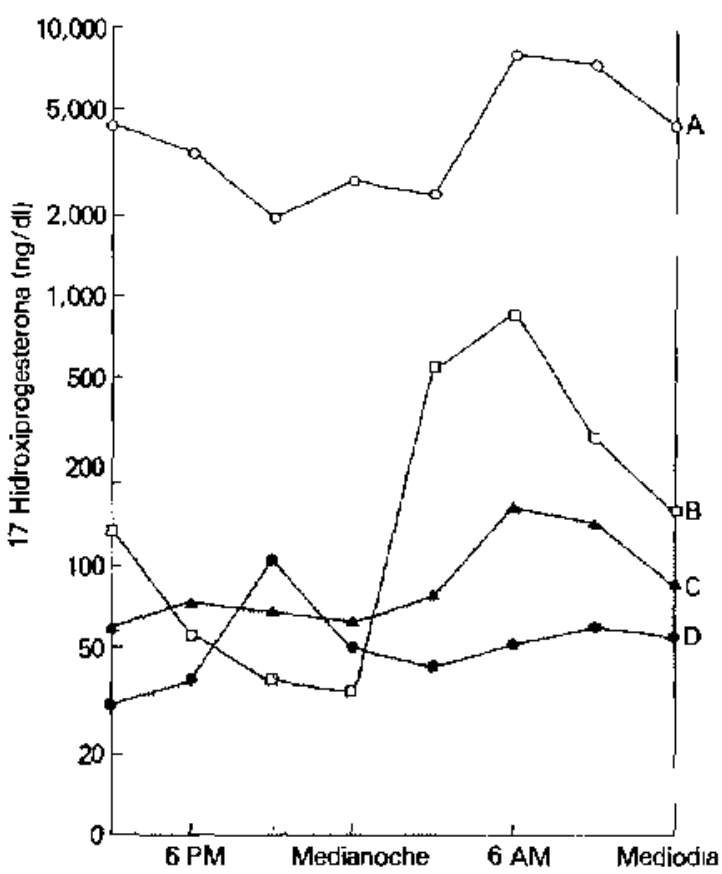

Figura 3. Concentraciones plasmáticas de 17 hidroxiprogesterona durante el tratamiento de 4 pacientes con el sindrome adrenogenital. Nótese la escala logirítmica en la ondenada. La zona stis representa el rango normal.

sa, de la sariedad no perdedora de sal. Este grupo de enfernos, formado por adolescentes de anbos sexos cuyas edades fluctuaron entre 13 y 18 años, estaba bajo tratamiento con hidrocortisona a una dosis entre 15 y $30 \mathrm{mg} / \mathrm{M}^{2} /$ día, dividida cada 8 horas.

El paciente A parece estar recibiendo una dosis insuficiente de glucocorticoide, cono se evidencia por los niveles elevados de 17 hidroxiprogesterona durante las 24 horas del estudio. Ello tue confirmado por una elevación de los 17 cetosteroides y el pregnanetriol, así como una aceleración del creciniento y maduración ósea. Posteriormente se comprohó que este adolescente no estaba recibiendo la dosis de hidrocortisona que se habja recetado.

El paciente B, bajo tratamiento en otra institución. consultó en nuestra clínica debido a crecimiento acelerado y niveles marginalmente elevados de pregnanetriol. Ello sugiríó la posibilidad de un tratarniento insuficiente, lo que hizo considerar un aumento en la dosis de hidrocortisona. Sin embargo, la determinación de niveles seriados de 17 hidroxiprogesterona demostró que este esteroide sólo estaba elevado en la madnugada. Por este motivo, se incrementó la proporción de 
hidrocortisona administrada en la noche, sin aumentar la dosis total diaria. Esta maniobra resultó en lá nomalización de los niveles matinales de 17 hidroxiprogesterona y del pregnanetriol. En otras palabras, las concentraciones seriadas de 17 hidroxiprogesterona permitieron definir las horas del día en que este paciente estaba mal controlado, y se pudo corregir este problema al aurnentar la dosis de glucocorticoide administrada en la noche. Esta maniobra terapéutica se logró sin aumentar la dosis total de hidrocortisona, lo que tiene importancia cuando se considera que en alguros pacientes, aun pequeños incrementos en las dosis de glucocortivoides, pueden conducir a retraso del crecimiento.

Los pacientes $C$ y D demuestran la inutilidad de la 17 hidroxiprogesterona para distinguir entre el paciente bien controlado y aquél tratado con una dosis excesiva de glucocorticoide. El paciente $\mathbf{C}$ tiene niveles normales de este esteroide que sugieren un tratamiento adecuado. Ello fue confirmado por la normalidad de sus metabolitos urinarios y de su desarrollo pondoestatural. El paciente $\mathbf{D}$ también tiene niveles normales de 17 hidroxiprogesterona, pero sufre de retraso del crecimiento lo que sugiere que está siendo tratado con una dosis excesiva de hidrocortisona. Estos enfermos sirven para enfatizar el concepto de que la 17 hidroxiprogesterond, tal cono los 17 cetosteroides y el pregnanetriol, no permite distinguir entre un tratamiento adecuado y uno excesivo.

Finalmente, es necesario memcionar que la 17 hidroxiprogesteronia tiene cierta utilidad en el control de la dosis de mineralocorticoides en los pacientes con el síndrome adrenogenital asociado con pérdida de sal. Algunos de estos enlenmos pueden tener concentraciones elevadas de $17 \mathrm{hi}$ droxiprogesterona y de renina plasmática, ‘ue sugieren una dosis insuficiente de mineralocorticoides. $23,59,50$ Este cuadro se puede corregir con un aumento en la dosis del esteroide retenedor de sal, lo que generalmente nornaliza los niveles de 17 hidroxiprogesterona y de renina plasmática. ${ }^{23}$, 54, En otras palabras, la 17 hidroxiprogesterona también pucde servir como indice del déficit de mineralocorticoides, presente en los pacientes perdedores de sal, iungue otros indices como la renina plasmática son de mayor utilidad en este sentido.

Como corolario se puede indicar que, auncue la 17 hidroxiprogesterona tiene utilidad en el tratamiento de los pacientes con el síndrome adreno- genital, esta determinación no ha reemplazado a los indices nás tradicionales de control terapéutico. En especial, los parámetros clínicos y los 17 cetosteroides y pregnanetriol, siguen teniendo mucha vigencia en el control de los pacientes pediátricos con este síndrome. La necesidad de obtener niveles seriados de 17 hidroxiprogesterona para tener una visión integrada de la función suprarrenal, con el costo que esto involucra, ha limitado la utilidad de esta determinación en el control de los enfermos con el síndrome adrenogenital.

\section{CONCLUSIONES}

El radioinnunoanálisis para la 17 hidroxiprogesterona representa un avance significativo en e] diagnóstico del síndrome adrenogenital. Estu determinación tiene particular utilidad durante el periodo neonatal, cuando las caracteristicas especiales del metabolismo suprarrenal pueden interferir con nuestra capacidad para establecer este diagnóstico basado en los niveles de 17 cetosteroides y pregnanetriol.

Los niveles de 17 hidroxiprogesterona durante la estimulación suprarrenal han pennitido diagsosticar un alto porcentaje de portadores heterocigotos, y numerosos pacientes con las formas críptica y adquirida del síndrome. La tipología HLA ha sido de gran valor en este sentido, y ha contribuido a la completa caracterización hormonal y genética de algunas familias con este síndrome. Nuestro concepto de la entidad clínica representada por el síndrome adrenogenital se ha expandido notablemente como resultado de estos estudios.

La 17 hidroxiprogesterona constituye un índice extremadamente sensible de la función suprarrenal en los pacientes con el síndrome adrenogenital por deficiencia de 21 hidroxilasa, y puede ser de utilidad en el control de estos enfermos. Sin embargo, los niveles de 17 hidroxiprogesterona deben ser analizados junto con otros parámetros, como son el desarrollo pondoestatural, la maduración ósea, y los 17 cetosteroides y pregnanetriol, para obtener un cuadro que retleje en forma fidedigna el grado de control terapéuticn de estos enfermos.

\section{AGRADECIMIENTOS}

El autor expresa sus agradecimientos a los Drs. 
Alfred M. Bongiovanni, George P. Chrousos y D. Lynn Loriaux por su valioso estímulo y colaboración en estos estudios.

\section{REFERENCIAS}

I Childs, B., Grambach, M.M. and Van WyK, JJ.- Vírilizing adrenal hyperplasia: a genetic and homonal study. J. Clin. Invest. $35: 213$, 1956.

2 Wilkirs, L., Lewis, R.A., Klein, K, et al.: Suppression of androgen secretion by cortisone in case of enggenital adrenis hyperplasia: preliminary report. Bull. Johns Hopkins Hosp. 86: $249,1950$.

${ }^{3}$ Ebertein, W.R. and Bongiovanni, A.M.: Plasma and urinary corticosteroids in hypertensive tom of congenital adrenal hyperplasia J. Biol. Chem. 223; 85, 1956.

4 Bangiovanni, A.M.: The adrenogenital syndrome with deficiency of $3_{\beta}$-hydroxysteroid dehydrogenase. J. Ciin. Invest. 41: 2086,1962 .

5 Biglieri, F.G., Herron, MA. and Brust, N.: 17-hydroxylation deficiency in man. J. Clin. Invest. 45: 1946, 1966.

6 Prader, A., and Gutener, H.P.; Das Syndrom des Pseudohemaphroditismus masc;ulinus bei Kongenitaler Nebennierenrinden hyperplasie ohne Audragenuber produktion. Helv. Paediatr. Acta, 10:397, 1955.

${ }^{7}$ Zachmann, M.J., Vellmin, A., Hamilton, W., et al. Steroid 17, 20 desinolase deficiency. Clin. Enducrinol. 1: 369, 1972.

8 Ulick, S., Gautier, E., Vetter, K.K, et at.: Ar aldosterotie biosynthetic defect in a salt losing disorder. J. Clin. Endocrinol. Metal. 24: 669, 1964.

9 Visser, H.K.A., and Cost, W.S.: A new hereditary defect in the hioss nthesis of aldosterone. Acta Endocrinol. 47: 589, 1964.

10) Bongiovanni, A.M., asd Hant, A.W.: The adrenongenital syn. drome. N. Engl. J. Med. 264: 1283, 1342, 1391, 1963.

11 Bryan, G.T. Kliman, B. and Bartter, F.C.: Inpaired aldosterone production in "salt lusing" congenital adrenal hyperplasia. J. Clin. Invest. 44: $457,1965$.

12 Kowarski, A., Finkelstein, J.W., Spaulding, J.S., et al.: Aldosterome secretion rate in congenital adrenal hyperplasia. A discussion of the theones on the pathogenesis of the saltlosing torm of the syndrome. J. Clin. Invest. 44: 1505, 1965.

1.3 Zimmermonn, W': E,ine Farbreaktion der sexual honnone und ilre Anwendung zur culantitativen culorimetrischen Bestmmung. Zt.schr. R. plyysidul. Chem. 233: 257, 1935.

14 Bongivanni, A.M., and Clayton, C.W.: Simplified methol for routine detemination of pregnanedisl and pregnanetriol in urine. Hull. Johns Hopkins Hosp. 94: 180, 1954

15 Bondicievanni, A.M.: Detection of preguandiol and pregniar trisl in urine of patients with adrenal hyperplasia: suppression with cortisone: prelininary report. Bult. Johns Hopkins Hosp. 92. 244, 1953.

16 Goldwan, A.S., Yakovac, W.C. and Bongiovanni, A.M.: Development of activity of 3 -hydroxysteroid dehydrogentase in hunatr fetsl tissues and in two anencephalic newbons. J. Clin. Endx-rinol, Metati. 26: 14, 1966.

1: Eberlein, W.R.: Steroids and sterols in umbulical cord blood. J. Cliti. Endoerinol. Metab. 25: 1101 , 1965.

1h Shackleton, C.H.L.: Congenital adrenal hyperplasia caused by defect in steroid 21-hydroxylase. Estublishment of definitive urinary steroid excretion pattent during first weeks of Jile. Clin. Chim. Actd 67: 287, 1976.

(5) Shuckleton, C.If., Mitchell, F.L. und Farquhard W.: Ditticulties in the diagnosis of the adrenogenital syndrome in infancy. Pediatrics 49: 198, 1972.

20 Abraham, C.E., Swerdloff, R.S., Tulchinsky, D., et al.: Radioinununoatsay of plasma 17 hydroxyprogesterone. J. Clin. Endocinol, . Vetal. 33: 42, 1971
21 Franks, A.C.: Screening tests for congenital adrenal hyperplasia. J. Clin. Endocrinol. Metab. 35: 831, 1972.

22 Bames, N.D., and Atherden, S.M.: Diagnosis of congenital udrenal hyperplasia by measurement of plusma 17-hydroxyprogyesterone. Arch, Dic. Child, 47: 62, 1972.

23 Iftughes, L.A, and Winter J.S.D.: The application of a serum $170 \mathrm{H}$-progesternne radioimmunoassay to the diagrosis and management of congenital adrenal hyperplesia. J. Pediatr. 88: $766,1976$.

24 Lippe, B.M., LaFranchi, S.H., Lavin, N., et al.. Serum 17-_hydmxyprogesterone, progesterone, estradiol and testosterone in the diagnosis and management of congenital adrenal hyperplasia. J. Pediatr. \&5: 785, 1974.

25 Levine, L.S., Dupont, B., Lorenzen, F., et al.: Genetic and hornonal characterization of cryptic 21-hydroxylase deticiency. J. Clin. Endocriuol. Metab. 53: 1192, 1981.

26 Nagumani, M., McDonough, P.G., Ellegood, J.O., et al.: Motemal and amniotic fluid $17_{\alpha}$-hydroryprogesterone levels duritg pregnancy: Diagrosis of congervital adrenal hyperplasla in utero. Am. J. Obstet. Gyт1ecol. 130: 791, 1978.

27 Pang. S., Murphey, W., Levine, L.S., et al.: A pilot newborn screening for congerital adrenal hyperplasia due to 2l-hydroxylase deficiency at New York Hospital and Alaska Abstract N. ${ }^{\circ}$ 44. First Joint meeting LWPES-ESPE, Geneva, Switzerland. 1981.

${ }^{2 \$}$ Pang, S., Hotchkiss, J, Drash, A.L., et al.: Mictofilter paper method for $17_{\alpha}$-hydroxyprogesterone radioimmunoassay: its applicution for rapid screening for congenital adrenal hyperplasia. J.Clin. Endocrimol. Metab. 45: 1003, 1977.

29 Blankstein, J., Fuimon, C., Reyes, F.I, et at: Adult-onset tamilial adrenal 21-hydroxylase deficiency, Ans. J. Med. 6甘: $44 \mathrm{I}, 1980$

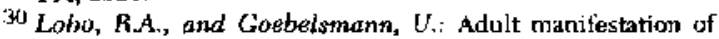
congenital adrenal hyperplasia due to incomplete 21-hydroxylase delicjency mimicking polycystic ovarian disease. Am. J. Obstet. Gynecol. 138: 720, 1980.

31 Chrotsos, C.P., Loriaux, D.L., Mann, D.Ln, et al. Late-anset 21-hydroxylase deficiency mimicking idiupathic hirsutism or jolycystic ovarias disease. Amn. Int, Med. 96: 143, 1982.

32 Chrousos, G.P., Loriaux. D.L., Sherins, RJ., et al.: Unilaterial testicular enlargement resulting tiom inapparent 2l-hydroxylase deficiency. J. Urol. 126: 127, 1981.

23 New, H.l., Lorenzen, F, Pang, S., et al.: "Acruired" adrenal hyperplasia with 21 -hydroxylase delichency is not the same genetic disorder as congenital adrenal hyperplasia. J. Clin. Endocrinol. Metab. 48: 356, 1979.

34 Migeon, C.J., Rosenwaks, Z., Lee, P.A., et al: The attenuated forn of congenital adrenal hyperplasia as an allelic form of 21-hydroxylase deficiency. J. Clin. Endocrinol. Metab. 5l: $647,1980$.

35 Yen. S.S.C. Chronic anovulation due to inappropriate feedback system. En Yen, S.S.C, and Jafiee, R.B. Eds, Reproductive Endlocrinology. Philadelphia, W.B., Saunders. 1978.

36 Gabriloce, J.L, Sharmo, E.C., and Dorfman, R.1.: Adrenocortical 11-Ise ta hydroxylase deficiency and virilism first manitest in the adult woman. N. Engl. J. Med. 272; 1189, 1965.

37 Rosenfield, H.L., Richo, B.H., Wolfstorf, J I., et al.: Pubertal presentation of congenital ${ }_{S}-3_{\beta}$-hydroxysteroid debydrogeuase deficiency. J. Clin. Endocrinol. Metab. 51: 345, 1980.

3 Songiovanni, A.M.: Acquired adrenal hyperplasia with special reference: to $3_{\beta}$-hydroxysteroid dehydrogenase. Fertil. Steril. 35: 599, 1981 .

3 Dupont, B., Oberfield, S.E., Smithwick, E.M., et at.: Close: getletic linkage between HLA and congenital adrenal hype ${ }^{-}$ plasia $(21$ hydroxylase deficiency). Lancet 2: 1344, 1977.

* Cleveland, W.W., Nikezic, M., and Migeon, C.J.: Response to 11 -hydroxylase inhibition (SU-4885) in males with adrenal hyperolasia and in their parents. J. Clin. Endocrinol. Metab. 22: 281.1962. 
41 Hall, R., Smith, PA., Haikness, R.A, et al. A stidy of the parents of patients with congenital adrenal hyperplasia: detection of the heterozygote. Price. Fusy. Sox. Med. 63: 1040, 1970.

${ }^{42}$ Qazi, Q.H., Hill,J.G, and Thompson, M.W.: Steroid studies in parents of paticrits with congenital virilizing adrenal hyperplasia. J. Clir. Endocrinol. Metab. 33: 23. 1971.

4.3 Lee, P.A., and Careis, F.J.: Evidence for partial 21-hydroxylase deliciency among heterozygnte carriers of comgenita] adrenal hyperplasia. J. Clin. Endoerinol. Metab. 41: 415, 1975.

44 Gitai,J.P. Kowarski, A.A., and Migeon, C.J.: The detection of heterozygmus edrrier for congenital virilizing adrenal hyperplasid. J. Pediatr. 90: 924, 1977.

45 Krensky, A.M., Bongiovorni, A.M., Marino, J, ef al.: Identification of heterozygote carriers of congenital adrenal hyperplasia by radioimmunosassay of serum $17-0 \mathrm{H}$ progesteroue. J. Pediatr. 90: 930, 1977.

${ }^{46}$ Cassonla, F, Tenore, A., Parks, J.S., et al.; Serum 21-deoxycontisol and 17-hydroxypregnenelone in parents of patients with congenital adrenal hyperplasia. J. Endixrinol. Invest 3: $13 \overrightarrow{7}, 1980$.

47 Levine, L.S., Zachmann, M., New, M.L., et al.; Genetic mapping of the 2l-hydroxylase defieiency gene within the HLA linkage group. N. Engl. J. Med. 299: 911, 1978.

48 Lorenzen, F, Pang, S., New, M.I., et al.: Honmonal phenotype and HLA-genotype in tamilies of patients with congenital alrenal hyperplasia (21-hydroxylase deficiency). Pediatr. Res. 13: 1356, 1979.

49 Sperling, M.A., Kentsy, F.M., Schutt-Aine, J.C., et al.: I, inear growth and growth hormonal responsiveness in treated congersital adrenal hyperplasia. Amer. J. Dis. Child. 129: 408, 1971 .

5t) Fappaport, R., Bouthreuil, E., Marti-Hennenberg, C., et al.: Linear growth rate, bone ruaturation and growth hurrosne secretion in prepubertal children with congenital atrenal hyperplasia. Actu Paediatr. Scand. 62: 513, 1973. 5l Kirkland, H.T., Keenan, E.S., Holcomb, J, $H_{+}$, et al.: The effect of therapy on mahuse height in congenital adrental hyperplusia. J. Clin. Endocrinol Metab. 47; 1320, 197 B.

52 Kopion, S.A.: Disorders of the adrenal cortex Il: Congenital adrenal hyperplasia. Pediatr. Clin, of North Ain. 26: 77, 1979.

${ }^{53}$ Bdiley, C.C., Komrower, G.H., Palmer, M.: Managenent of congerital adrenal hyperplasia. Unuary sterad estimations review of the ir value. Arch. Dis. Child. 53: 132, 1978.

54 Golden, M.P., Lippe, B.M., Koplan, S.A., et al.:Management of congenital adrenal hyperplasia using serum dehydroepianIrosterone sulfate and 17-hydroxyprogesterone concentrations. Pediatrics 61: 867, 1978.

${ }^{55}$ Hughes, 1.A., and Winter, J.S.D.; The relationships between serum concentrations of 17 OH progesterme and other serum and urinary steroids in patients with congenital adrenal hyperplasia. J. Clin. Endocrinol. Metab, 46; $48,1978$.

56 Grant, D.B., Dillon, M.J., Atherden, S.M., et al.: Congenital adrenal hyperplasia: renin and sterrid values dhuing trealment. Evт. J. Pediatr, 126: 89, 1977.

${ }^{57}$ Frisch, H., Parth, K., Schober, E., et ol.: Circadian pattenis of plasma cortisol, 17-hydroxyprogesterone, and testosterone in cormenital adrenal hyperplasja. Arch. Dis. Child. 56: 208, 1981.

58 Kunth-Schutz, S., Virdis, R., Saenger, P., et ol.: Serum androgens as a continuing index of allequacy of treatonent of con. genital adrental hyperplasja. J. Clin. Endocrinol. Metab. 46: $4 \overline{3} 2,1978$.

59 Hughes, I.A., Wilton, A., Lole, C.A., et al.: Cuntimuing need tor mineralocorticoid therapy in salt losing congenital adtrenal hyperplasia. Arch. Dis. Child. 54: 350, 1979.

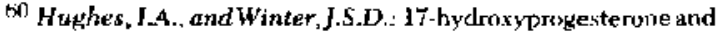
plasma renin activity in congenital adsenal hyperplasia. En Lee, P.A., Plotrick, L., Kowarski, A.A., and Migeon, C. J., Eds. Corgenital arlrenal hyperplasia. Baltimore. Unjversity Päk Press, 1977. 